\title{
External application of traditional Chinese medicine in the treatment of bone cancer pain: a meta-analysis
}

\author{
Yan Xiangyong ${ }^{1} \cdot$ Yan Zhongsheng $^{1} \cdot$ Liu Wenchao $^{2}$ - Ding Hui ${ }^{1}$ - Qiao Shuzhou ${ }^{3}$. \\ Chen Gang ${ }^{4} \cdot$ Wang XiaoHui ${ }^{5}$ Zhang Lian ${ }^{6}$
}

Received: 5 December 2014 / Accepted: 6 April 2015 /Published online: 19 April 2015

(C) The Author(s) 2015. This article is published with open access at Springerlink.com

\begin{abstract}
Background Bone cancer pain presents a clinical challenge with limitations of current treatments. Many patients seek additional therapies that may relieve pain. Many external applications of traditional Chinese medicines (EAs-TCMs) have been evaluated in clinical trials, but fewer are known about them outside of China. The objective of this study is to assess the efficacy for bone cancer pain.

Methods A systematic literature search was conducted in seven databases until December 2014 to identify randomized controlled trials (RCTs) about EAs-TCMs in the treatment of bone cancer pain. The primary outcome was total pain relief rate. The secondary outcomes were adverse events at the end of treatment course. The methodological quality of RCTs was assessed independently using six-item criteria according to the Cochrane Collaboration. All data were analyzed using Review Manager 5.2.0. We included any RCTs evaluating an EA-TCM for the treatment of bone cancer pain. We conducted a meta-analysis.
\end{abstract}

Yan Xiangyong

yanxiangyong@126.com

1 Department of Integrative Medicine, Lanzhou General Hospital of the People's Liberation Army, Lanzhou 730050, China

2 Department of Oncology, Xijing Hospital, The Fourth Military Medical University, Xi'an 710032, China

3 Senior Cadres Ward I, Lanzhou General Hospital of the People's Liberation Army, Lanzhou 730050, China

4 Department of MR, Lanzhou General Hospital of the People's Liberation Army, Lanzhou 730050, China

5 Experimental Center of Medical, Lanzhou General Hospital of the People's Liberation Army, Lanzhou 730050, China

6 Department of Orthopaedics, Lanzhou General Hospital of the People's Liberation Army, Lanzhou 730050, China
Results We included six RCTs with 534 patients. In general, the reporting of methodological issues was poor. Compared with morphine sulfate sustained release tablets (MSSRTs) or radiotherapy or bisphosphonates, we analyzed data from five trials reporting on complete response effect score (relative risk $(\mathrm{RR})=5.38,95 \%$ confidence interval $(\mathrm{CI})=2.80-10.31$, $P<0.00001)$ and partial response $(\mathrm{RR}=1.18,95 \% \mathrm{CI}=$ $1.02-1.37, P=0.02)$ and six trials reporting on total pain relief rate $(\mathrm{RR}=1.49,95 \% \mathrm{CI}=1.43-1.67, P<0.00001)$. Six $\mathrm{RCTs}$ showed significant effects of EA-TCM for improving pain relief in patients with bone cancer pain. In addition, no severe adverse events were found.

Conclusion This systematic review showed positive but weak evidence of EA-TCM for bone cancer pain because of the poor methodological quality and the small quantity of the included trials. Future rigorously designed RCTs are required.

Keywords External application of traditional Chinese medicines $\cdot$ Bone cancer pain $\cdot$ Clinical trial

\section{Introduction}

Bone pain is a common type of cancer pain, cancer-induced bone pain is a common symptom in cancer patients, and spontaneous breakthrough pain is severe and difficult to control [1], which strongly affect the patients' quality of life. Tumor-derived, inflammatory, and neuropathic factors may simultaneously contribute to cancer pain, such as bone cancer pain [2]. Bone metastasis can occur in advanced cancer in up to $70 \%$ of patients with advanced breast or prostate cancer and in approximately $40 \%$ of patients with lung, kidney, or thyroid cancer [3]. The underlying pathomechanism of bone cancer pain is largely unknown. Bone destruction, reactive muscle spasm, increased local and blood concentration of 
calcium ions, and the release of inflammatory mediators by tumor cells are all implicated in the pathomechanism [4]. Tumor causes severe disintegration of the cortical and trabecular bone. This inevitably leads to fractures at a later stage, an occurrence seen in approximately $50 \%$ of patients with bone metastases [5].

Owing to the significant health risk of bone cancer pain and the limitations of currently available conventional therapies, patients have been suffered great pain, but in China, traditional Chinese medicine (TCM) has been used to alleviate cancer pain for more than 2000 years. In recent decades, unprecedented attention has been attached to external application of traditional Chinese medicine (EA-TCM) in modern time due to its potential efficacy on bone cancer pain. EAs-TCMs have been widely used in bone cancer pain patients in both Western medicine hospitals and TCM hospitals. As an important complementary therapy for bone cancer pain, TCM has gradually shown its typical curative effect. EA-TCM is a commonly employed complementary therapy for improving the patients' quality of life for bone cancer pain patients. It has provided an important complement or alternative to the current health care in many countries and has been increasing in popularity. Now, there are many clinical trials of EA-TCM for bone cancer pains that have been reported with positive results. However, there is no minutely evaluated evidence and meta-analyses on the potential benefit of clinical herbs for bone cancer pain patients to justify their recommendation and their clinical role.

\section{Methods}

\section{Search strategy}

Seven databases were searched from their inception to December 2014. These included MEDLINE, Embase, and Cochrane Library as well as four Chinese medical databases: China National Knowledge Infrastructure database, VIP database for Chinese Technical Periodicals, Chinese Biomedical Literature database, and Wanfang database; we collect all randomized controlled trials from bone cancer pain patients. Search terms include "bone cancer pain," "metastatic bone," "metastatic," "external treatment," "external application," "traditional Chinese medicine," "Chinese traditional," "herbal medicine," "Chinese herbal," "randomized controlled trial," and "clinical trial" used individually or combined. The reference list of papers authenticated were scanned and retrieved for checking of relevance. All the searching was updated to December 2014.

\section{Study selection and data collection}

The three authors of this article managed the literature search, paper selection, and data extraction independently. Studies were selected by two independent reviewers according to the predetermined inclusion criteria. Disagreement was resolved by a third reviewer. The extracted data included title, authors, year of publication, age and number of participants, malefemale ratio, study size, diagnosis criteria, details of methodology, name of EA-TCM, specifics of the control interventions, treatment, outcomes, and adverse reaction for every research. Risk of bias was assessed in accordance with Cochrane 5.1.0 handbook of systematic reviews of interventions [6].

Quality assessment of the included randomized, controlled trials included sequence generation, allocation concealment, blinding of participant personnel and outcome assessors, incomplete outcome data, selective outcome reporting, and other sources of biases [7]. The quality of all included studies was categorized as C (low)/B (unclear)/A (high) risk of bias.

\section{Data analysis}

Data analysis was performed using the statistical package RevMan 5.2.0 software from the Cochrane Collaboration. Meta-analysis was performed only on studies that have acceptable homogeneity in respect of study design, controls, interventions, participants, and outcome measures. Dichotomous data were presented as relative risk (RR) and continuous outcomes as mean difference (MD), both with $95 \%$ confidence intervals (CIs). Statistical heterogeneity was tested by examining $I^{2}$, where $I^{2}<25 \%$ is considered as low heterogeneity, $25 \% \leq I^{2} \leq 50 \%$ is moderate heterogeneity, and $I^{2}>50 \%$ is high heterogeneity [8]. $I^{2}>50 \%$ or a $P$ value $<0.1$ indicates the possibility of statistical heterogeneity. The random effect model was used for the meta-analysis if there was significant heterogeneity, while the fixed effect model was used when the heterogeneity was not significant. Publication bias was explored via a funnel plot analysis.

\section{Results}

\section{Search results}

We identified 439 potentially relevant articles after duplicates were removed. Through screening titles and abstracts, 351 were excluded because they were not relevant or they are reviews, non-randomized control trials, and other treatment modalities. We conducted full-text evaluation for the remaining 88 articles, and 82 articles were excluded for not meeting our inclusion criteria. Among them, 45 articles designee to detect cost different and 30 trials have no enough outcomes. Seven articles are not about bone cancer pain. Finally, six studies [10-15], involving a total of 534 participants, met our inclusion criteria. 


\section{Study characteristics}

We searched primarily from the databases, with 690 citations screened. Because of evident ineligibility, the great majority of citations were excluded; finally, full-text papers of seven studies were included. A flowchart describing the searching strategy is shown in Fig. 1. The six studies included 534 patients in total (266 were in the control group, and 268 were in the treatment group). All studies were conducted in China, published between 2008 and 2014. One trial [11] compared EATCM with pamidronate disodium (PD) to PD individually. Another trial [14] compared EA-TCM with radiotherapy to radiotherapy individually. Four trials $[10,12,13,15]$ compared EA-TCM with morphine sulfate sustained release tablets (MSSRTs) to MSSRT individually. Detailed information of the seven studies is shown in Table 1.

\section{Assessment of the quality of methodology}

Most of the included studies were assessed to be poor methodologically, with Cochrane scores of B and C. All of the included studies mentioned randomization, but only two studies $[10,14]$ reported the method of random sequence generation. Insufficient information was provided to judge whether or not it was regulated properly. One study [10] mentioned allocation concealment but was not described. Double blindness was found in one randomized controlled trial (RCT) [10] in all the studies. Two trials $[10,14]$ reported the loss to follow-up, intended to analyze the reason. No studies used intention-to-treat (ITT) analysis. In general, one RCT [10] was deemed to have an unclear risk of bias and five RCTs [11-15] were deemed to have a high risk of bias based on the Cochrane risk of bias tool. The quality assessment of included studies is listed in Table 2.

\section{Clinical outcomes}

\section{Complete response}

We analyzed data from 5 trials, and complete response pain relief rate of EA-TCM in treating bone cancer pain patients was reported in 5 studies [10-12, 14, 15], including 493 patients. Applying meta-analysis, we found that products containing EA-TCM had a larger treatment effect than without EA-TCM. Results of meta-analysis suggested that patients who received EA-TCM treatment showed better treatment effect outcomes than the control group $(Z=5.08, \mathrm{RR}=5.38$, $95 \% \mathrm{CI}=2.81-10.31, P<0.00001$ ) (Fig. 2).

\section{Partial response}

We Pooled data from five trials $[10-12,14,15]$ reporting on PR between groups. The combined effects of five independent trial results showed that EA-TCM had improved partial response rate in patients with bone cancer pain when compared with MSSRT or bisphosphonate control individual. The pooled RR is 1.18 (95\% CI=1.02-1.37, $Z=2.28 P=0.02)$ (Fig. 2).

\section{Total pain relief rate}

All six studies adopted the total pain relief rate to assess the clinical improvement. Total pain relief rate of EA-TCM in
Fig. 1 Flowchart showing the identification of studies included in the review

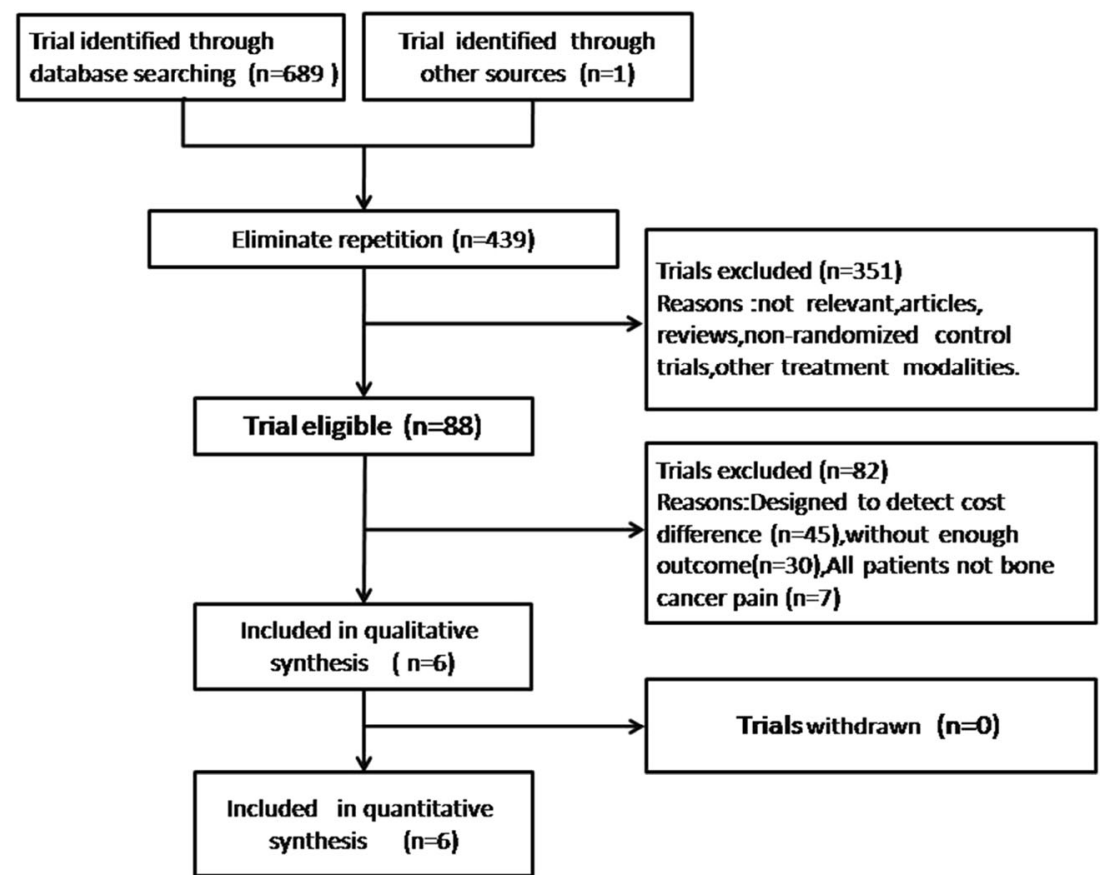




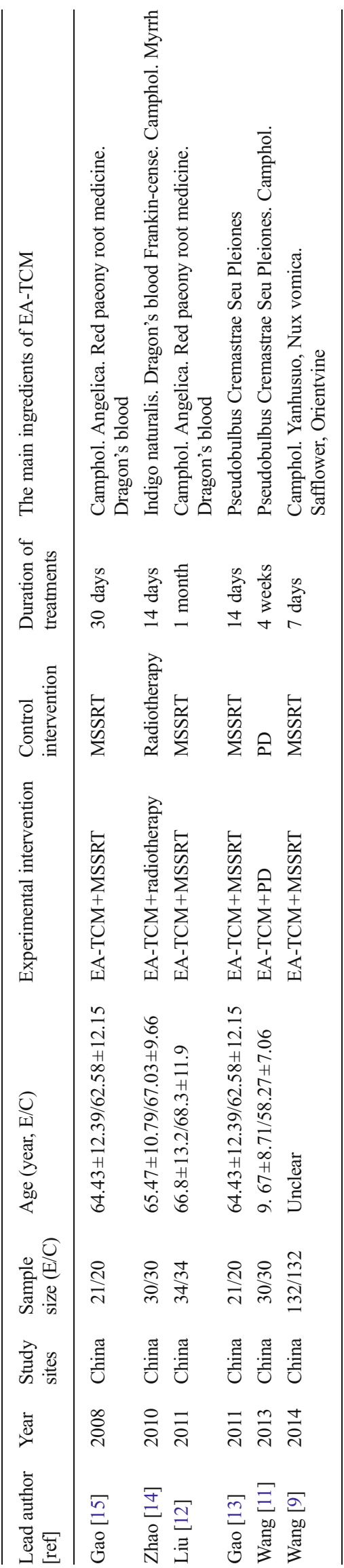

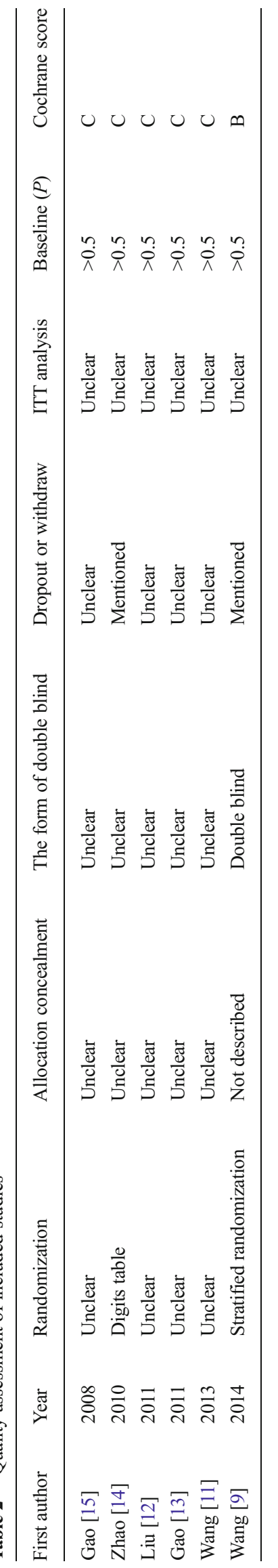


treating bone cancer pain patients was reported in 6 studies [10-15] including 534 patients. We found results of metaanalysis suggesting that patients who received EA-TCM treatment postoperatively could increase analgesic effect $(Z=6.83$, $\mathrm{RR}=1.49,95 \% \mathrm{CI}=1.43-1.67, P<0.00001$ ) (Fig. 2). No significant funnel plot asymmetry based on visual inspection was found, suggesting no evidence of publication bias (Fig. 3).

\section{Sensitivity analysis}

In the six included trials, there are two studies $[13,15]$ showing the small sample sizes. The sensitivity analysis showed that with low-quality trials precluded, the summary of RR and $95 \% \mathrm{CI}$ for the above effects remains the same (Table 3), indicating that the conclusions in our study are believable and trustworthy.

\section{Discussion}

In recent years, TCM is considered an important complementary therapy with beneficial effects for cancer patients, the

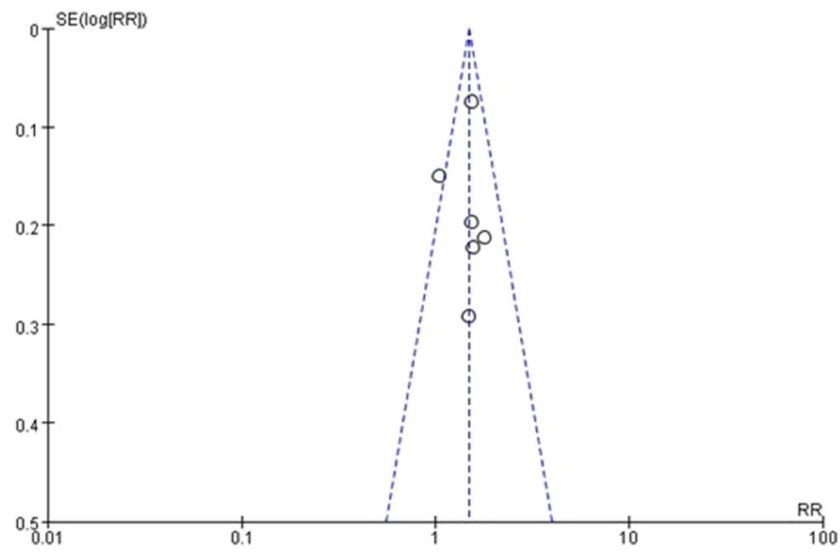

Fig. 3 Funnel plot for checking publication bias

holistic concepts of TCM that consider human beings as an integral part of nature, and any clinical presentation is an overall reflection of the whole human body that expands the horizon of modern medicine. Besides, during the long history of TCM, ancient physicians accumulated a tremendous amount of knowledge and experiences in treating human diseases.

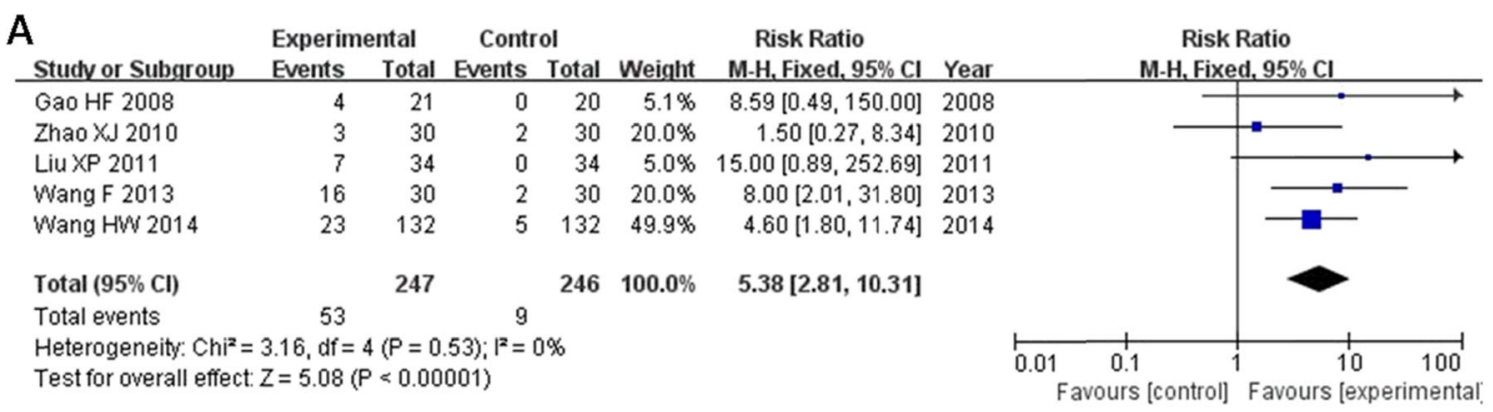

B

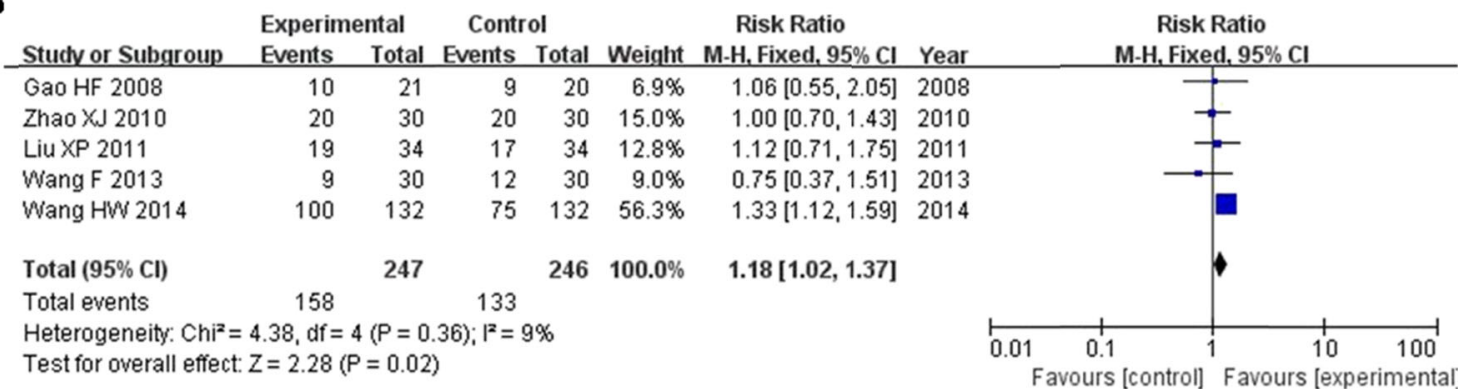

C

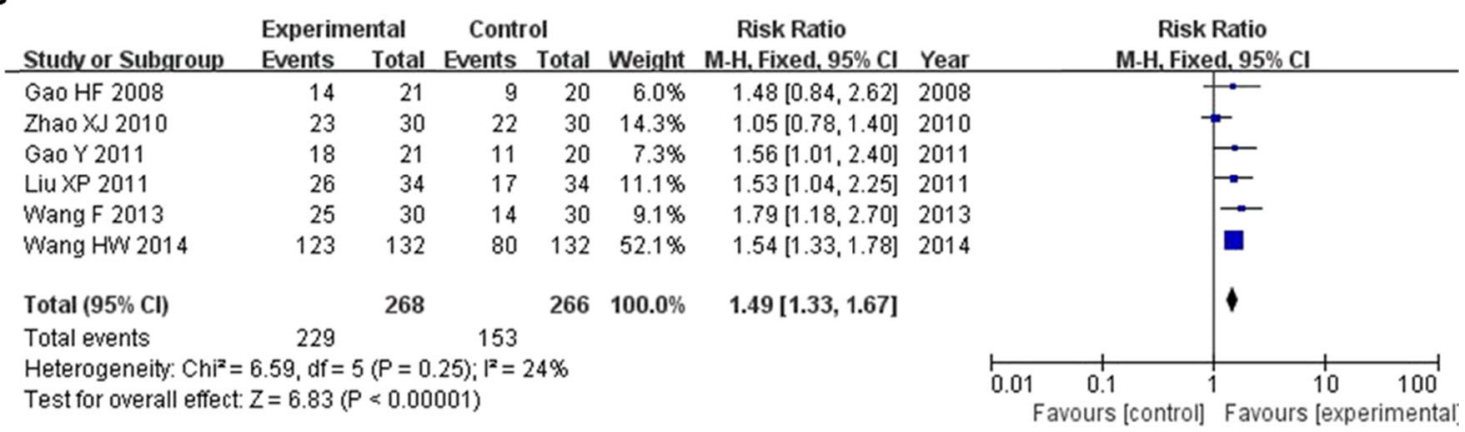

Fig. 2 a Forest plot of complete response. b Forest plot of partial response. c Forest plot of comparison of total pain relief rate 
Table 3 Sensitivity analysis

\begin{tabular}{lllll}
\hline First author & Index & Before exclusion & After exclusion & Statistical significance \\
\hline Gao [15] & $\mathrm{CR}$ & $\mathrm{RR}=1.49,95 \% \mathrm{CI}=1.33-1.67$, & $\mathrm{RR}=1.56,95 \% \mathrm{CI}=1.38-1.77$, & No difference \\
& & $Z=6.83, P<0.00001$ & $Z=7.07, P<0.00001$ & No difference \\
Zhao [14] & $\mathrm{RR}$ & $\mathrm{RR}=5.38,95 \% \mathrm{CI}=2.81-10.31$, & $\mathrm{RR}=5.21,95 \% \mathrm{CI}=2.67-10.16$, & $Z=4.85, P<0.00001$ \\
& & $Z=5.08, P<0.00001$ & & \\
& & & &
\end{tabular}

Although people increasingly use TCM, there are few metaanalyses to systemically review the clinical therapeutic effects of TCM. In addition, a large number of evidence shall possibly be missed if the literature search is restricted to Englishonly sources [16].

Whether it is morphine or radiotherapy or other methods of treatment of bone cancer pain, there were many adverse reactions: the primary adverse reactions were nausea and vomiting related to morphine [17], radiotherapy have many complications, and bisphosphonates have an influence on sclerotin [18]. If the financial burden to the patient is increased, finding a new cost-effective method of treatment is necessary. Some clinical studies have shown that topical medicine can reduce the amount of medication and adverse reactions [19, 20]. In China, TCM is in low price; it is very common in a pharmacy of grassroot health institutions. So, it is convenient and cheap. EA-TCM is a good choice for bone cancer pain patients. We supervised this meta-analysis in bone cancer pain patients to evaluate the clinical efficacy of EA-TCM as a complementary therapy from mainly Chinese-sourced studies. Although the quality of the studies is poorly satisfactory, we believed that EA-TCM could help improve analgesia treatment efficacy with MSSRT. There were fewer side effects in the treatment groups, and none of the effects was severe; only one study [14] mentioned the adverse effects of EA-TCM in which one patient has slight rash. No patients dropped out of their test trial due to the side effects of EA-TCM, which indicated that EA-TCM is safe for clinical use. TCM provides a beneficial effect in the treatment bone cancer pain patients.

Limitations of our study include the underlying concern about the quality of the included studies. Only positive trials are published in Chinese medical journals, and these trials reported are randomized; in fact, we do not determine that they are randomized. A recent evaluation by $\mathrm{Wu}$ et al. found that there were many studies labeled as RCTs with Chinese journals [21], and randomization is necessary to avoid selection bias. However, only two studies [10, 14] provided specific information on how the random allocation was generated. None of the included trials reported the allocation concealment; the common use of clinical efficacy rate as an ancillary outcome measure through subjective qualitative scores such as clinical cure, markedly effective, effective, and ineffective in Chinese is not internationally recognized; and the validity and reliability of that was uncertain in assessing the outcome.
Almost all the included RCTs claimed that the positive effect of EA-TCM combined with MSSRT or radiotherapy or bisphosphonates is better than that of MSSRT or radiotherapy or bisphosphonates alone. Negative findings almost have not been reported. We tried to conduct extensive searches for unpublished material, but no unpublished negative studies were found. Therefore, we could not exclude the possibility that studies with negative findings remain unpublished. We examined publication bias through both visual inspection of the funnel plot on the primary outcome (RR) and through statistical tests, but these were unable to identify publication bias. However, funnel plots cannot rule out publication bias and we remain cautious that many negative trials likely exist.

\section{Conclusion}

In summary, the EA-TCM appears to be able to improve total pain relief; however, current evidence is insufficient to support the efficacy of EA-TCM for bone cancer pain patients. Because the included studies were of generally poor quality and had small sample sizes, although limited by the study quality, TCM may provide beneficial effect for bone cancer pain patients. Most rigorously controlled clinical trials would be preferred for future study.

Conflict of interest The authors declared that they have no conflicts of interest to this work.

Open Access This article is distributed under the terms of the Creative Commons Attribution-NonCommercial 4.0 International License (http:// creativecommons.org/licenses/by-nc/4.0/), which permits any noncommercial use, distribution, and reproduction in any medium, provided you give appropriate credit to the original author(s) and the source, provide a link to the Creative Commons license, and indicate if changes were made.

\section{References}

1. Paley CA, Bennett MI, Johnson MI (2011) Acupuncture for cancerinduced bone pain? Evid Based Complement Alternat Med 2011: 671043

2. Peters CM, Ghilardi JR, Keyser CP, Kubota K, Lindsay TH et al (2005) Tumor-induced injury of primary afferent sensory nerve fibers in bone cancer pain. Exp Neurol 193:85-100 
3. Coleman RE (2006) Clinical features of metastatic bone disease and risk of skeletal morbidity. Clin Cancer Res 12:6243s-6249s

4. Ripamonti C, Fulfaro F (2000) Malignant bone pain: pathophysiology and treatments. Curr Rev Pain 4:187-196

5. Rubens RD (1998) Bone metastases - the clinical problem. Eur J Cancer 34:210-213

6. Higgins JPT, Green S (eds)(2011) Cochrane handbook for systematic reviews of interventions, version5.1.0 (updated September 2011). The Cochrane Collaboration, available from www. cochranehandbook.org

7. Schneider V, Levesque LE, Zhang B, Hutchinson T, Brophy JM (2006) Association of selective and conventional nonsteroidal antiinflammatory drugs with acute renal failure: a population-based, nested case-control analysis. Am J Epidemiol 164:881-889

8. Higgins JP, Thompson SG (2002) Quantifying heterogeneity in a meta-analysis. Stat Med 21:1539-1558

9. Wang H, Wang W, Yu M et al (2014) Clinical research on TCM external treatment combined with morphine in treatment of the pain of bone metastases. Chin Arch Tradit Chin Med 29:3018-3021

10. Liu Y (2013) Acupoint application on morphine sulfate sustainedrelease tablets in the treatment of bone metastases pain. Chin Community Doct 15:218-219

11. Wang F, Fen L, Zhang P, Gao Y (2013) Clinical observation on reliving pain in bone metastasis patients by pamidronate disodium combined with Bushen Huayu Chinese medicine. Chin J Exp Tradit Med Formulae 19:327-329

12. Liu X (2011) Clinical research on external application of Chinese medicine combined with morphine sulfate sustained release tablets in the treatment of cancerous pain of metastatic tumor of bone. China Med Her 8:107-108

13. Gao Y, Feng L (2011) Clinical observation on metastatic bone pain treated with application therapy with Pseudobulbus Cremastrae Seu Pleiones. World J Integr Tradit West Med 06:574-576

14. Zhao X (2010) The clinical efficacy of Xiao Zhen Zhi Tong formula for external use combined with radiotherapy [master's thesis]. Beijing University of Chinese Medical

15. Gao H, Hou J, Zhang H, Hu Y, Zhou L (2008) Clinical efficacy of external medical herbs combining morphine sulfate on metastatic bone tumor pains. Shanghai J Tradit Chin Med 42:27-29

16. Ezzo J, Berman BM, Vickers AJ, Linde K (1998) Complementary medicine and the Cochrane Collaboration. JAMA 280:1628-1630

17. Hong D, Flood P, Diaz G (2008) The side effects of morphine and hydromorphone patient-controlled analgesia. Anesth Analg 107: 1384-1389

18. Xie S, Zhan L, Pan W (2011) Progress of zoledronic acid adverse reactions mandibular bone and correlation. J Moden Stomato 25: $460-462$

19. Fen Y, Cui Y, Xu R, Jiang M (2014) Study progression in treating bone-transfer cancer by traditional Chinese medicine. Glob Tradit Chin Med 7:652-655

20. Chen MX, Huang LZ, He YH, Zhang H, Cao JX (2011) A clinical study on the analgesic effect of compound Shanshu powder on cancerous pain used in a way of external application: a report of 60 cases. Hunan Zhong Yi Xue Yuan Xue Bao 24:37-39

21. Wu T, Li Y, Bian Z, Liu G, Moher D (2009) Randomized trials published in some Chinese journals: how many are randomized? Trials 10:46 